\title{
¿Crisis febriles complejas o síndrome de Dravet? Descripción de 3 casos clínicos
}

\author{
M. Hernández • M. Pedraza ・T. Mesa ・M. Troncoso
}

\begin{abstract}
Complex febrile Seizures or Dravet syndrome? Description of 3 case reports
\end{abstract}

Introduction: Dravet syndrome (DS) is one of the most intractable forms of epilepsy that begins in infancy. This syndrome is characterized by beginning with complex febrile seizures (FS) in a healthy infant and progresses to refractory epilepsy with psychomotor regression. The detection of a SCN1A mutation encoding the sodium channel can confirm the diagnosis. Objective: To report 3 confirmed cases of genetically DS. Case reports: We describe 3 girls diagnosed with complex FS that started when they were between 2 and 7 months old. FS were frequent, hemi generalized and myoclonic associated with recurrent febrile status epilepticus (SE). Despite FS and SE recurrence, the psychomotor development, electrophysiological studies and magnetic resonance imaging (MRI) of the brain were normal. After a year, they developed afebrile seizures progressing to refractory epilepsy with developmental regression. A molecular study detected SCN1A mutation confirming DS. The specific antiepileptic treatment and prevention of febrile episodes allowed partial control of epilepsy with some recovery of psychomotor skills. Conclusions: The high frequency complex FS associated with recurrent SE in a previously healthy infant should alert about the possibility of DS. Molecular diagnostics helps us to establish a drugs and non-drug therapies treatment, as well as long-term prognosis and genetic counseling.

(Key words: Dravet syndrome, febrile seizures, status epilepticus, severe myoclonic epilepsy in infancy, SCN1A).

Rev Chil Pediatr 2014; 85 (5): 588-593

\section{RESUMEN}

Introducción: El Síndrome de Dravet (SD) es una de las formas más intratables de epilepsia que debuta en lactantes con convulsiones febriles (CF) complejas recurrentes que evolucionan posteriormente a epilepsia

Recibido el 16 de mayo de 2014, última versión aceptada el 10 de octubre de 2014.

Marta Hernández Chávez $(\bowtie)$, Tomás Mesa Latorre

Neuropediatra, Sección Neurología Pediátrica, División de Pediatría, Facultad de Medicina. Pontificia Universidad Católica de Chile.

E-mail: mhernand@med.puc.cl

Mariela Pedraza Herrera

Residente Unidad Cuidados Intensivos Pediátricos, Clínica Dávila.

Mónica Troncoso Schifferli

Neuropediatra, Hospital Clínico San Borja Arriarán. 
refractaria con regresión psicomotora. La detección de una mutación del canal de Sodio (SCN1A) permite certificar el diagnóstico. Objetivo: Reportar 3 casos de SD confirmados genéticamente. Casos clínicos: Se describen 3 niñas con diagnóstico de CF complejas iniciadas entre los 2 y 7 meses de edad. Las CF eran frecuentes, hemigeneralizadas, mioclónicas asociadas a status epilepticus (SE) febriles recurrentes. A pesar de la recurrencia de CF y SE, tanto el desarrollo psicomotor como los estudios electrofisiológicos y la resonancia magnética (RM) cerebral, fueron normales. Posterior al año iniciaron crisis afebriles que evolucionaron a epilepsia refractaria con regresión del desarrollo. El estudio molecular detectó la mutación SCN1A confirmando SD. El tratamiento antiepiléptico específico y la prevención de cuadros febriles permitieron un control parcial de la epilepsia con recuperación de algunas habilidades psicomotoras. Conclusiones: La alta frecuencia de CF complejas asociadas a $S E$ recurrentes en un lactante previamente sano, debe alertar sobre la posibilidad de un $\mathrm{SD}$. El diagnóstico molecular nos permite instaurar un tratamiento antiepiléptico y terapias no farmacológicas además de un pronóstico a largo plazo y consejería genética.

(Palabras clave: Síndrome de Dravet, convulsiones febriles, status epilepticus, epilepsia refractaria, SCN1A). Rev Chil Pediatr 2014; 85 (5): 588-593

\section{Introducción}

El síndrome de Dravet (SD) descrito por Dravet en $1978^{1,2}$ tiene una incidencia estimada 1 por 40.000 niños $^{3,4}$. Se inicia en el primer año de vida con una historia de convulsiones febriles (CF) o convulsiones gatilladas por fiebre, cuadros infecciosos, aumento de la temperatura corporal (baños calientes) o estimulación fótica. Las CF son "complejas" (focales, hemiclónicas,prolongadas o Status epilepticus $(S E)$ febriles) o tónico clónico generalizadas. Después del primer año de vida evolucionan a convulsiones afebriles, $S E$ afebriles y ausencias atípicas. El retraso en el diagnóstico es frecuente porque a pesar de la recurrencia de CF y $S E$ febriles el desarrollo psicomotor (DSM) es normal al igual que el electroencefalograma (EEG) y las neuroimágenes antes del año de edad ${ }^{4,5}$. Entre el 1-4 año de vida hay una regresión del desarrollo con una discapacidad de leve a grave y epilepsia de difícil control evolucionando posteriormente a un período secuelar ${ }^{4,6}$.

Las CF afectan al 3-5\% de los menores de 5 años ${ }^{7}$. La National Institute of Health (NIH) las define como aquellas que se presentan entre el 3 mes y los 5 años, asociado a episodios febriles, sin evidencia de infección intracraneal o causa definida ${ }^{8}$. La Liga Internacional contra la Epilepsia (ILAE) incluye a lactantes mayores de 1 mes, sin antecedentes de crisis neonatales, crisis agudas sintomáticas u otro tipo de epilepsia9. Ambas definiciones son iguales, a excepción del rango mínimo de edad. Por la frecuencia de $\mathrm{CF}$, este es el primer diagnóstico que reciben los lactantes con $\mathrm{SD}$.

La subunidad alfa 1 del canal de sodio voltaje gatillado (SCNA1 o Sodium channel neuronal type 1 $\alpha$ subunit) (OMIM\# 182389) es el canal más relevante y con el mayor número de mutaciones relacionadas con epilepsia. Más del 70\% de los casos de Síndrome de Dravet (SD) o epilepsia mioclónica severa de la infancia $^{10}$ está asociada a una mutación del gen SCN1A y si adicionamos el "SD borderline" y "la epilepsia intratable de la infancia con crisis tónico clónicas generalizadas" este porcentaje es alrededor del $90 \%{ }^{11}$.

Se presentan 3 niñas con diagnóstico inicial de CF cuya clínica sugirió un SD confirmándose una mutación del SCN1A. El propósito es reportar características clínicas del Síndrome de Dravet, cuyo diagnóstico está basado en un estudio genético, y realizar una actualización en el tema.

\section{Casos clínicos}

\section{Caso 1}

Segunda hija, padres jóvenes no consanguíneos, primer hijo fallecido por una "meningitis", sin datos adicionales. Sin antecedentes de epilepsia en familiares de primer grado. Sin morbilidad perinatal. Se evaluó por primera 
vez a los 18 meses de edad, por falla hepática aguda asociada a ácido valproico.

A los 4 meses inició "CF complejas" tratadas con fenobarbital. Al año había experimentado 5 episodios de Status epilepticus febriles y múltiples $\mathrm{CF}$ prolongadas, mioclónicas, hemigeneralizadas. Su DSM fue normal hasta el año de vida al igual que electroencefalograma (EEG) standard y la Resonancia Magnética (RM) cerebral. Posterior al año inició una epilepsia refractaria con convulsiones y $S E$ febriles y afebriles asociado a regresión de habilidades psicomotoras adquiridas (marcha independiente y lenguaje).

En su examen destacaba ictericia, microcefalia (circunferencia craneana bajo percentil 2 de las curvas de Nelhaus), ausencia de marcha independiente y retraso de lenguaje expresivo y comprensivo.

El monitoreo vídeo-EEG identificó crisis eléctricas focales con actividad interictal frontal bilateral sincrónica. Los estudios metabólicos (ácidos orgánicos, aminoácidos en sangre y líquido céfalo raquídeo (LCR) y la microscopía electrónica de piel fueron normales.

El estudio molecular del gen SCN1A detectó la mutación c.3457G $>\mathrm{T}$ (p.Glu1153X) en estado heterocigoto en el exón 17, confirmando un SD. El estudio molecular de ambos padres fue normal.

La epilepsia se controló parcialmente con topiramato, Sulthiame y lacosamida, previamente había usado fenobarbital, topiramato y clobazam.

Por el estudio de deglución anormal asociado a neumonías frecuentes se realizó gastrostomía, que disminuyó la frecuencia de cuadros febriles agudos con disminución de $S E$. Su último control a los 6 años, con tratamiento triasociado, mostró una niña vigil, con lenguaje comunicativo y marcha independiente , Sin $S E$ desde los 2 años con una frecuencia de crisis de que disminuyó a 1-3 por semana de segundos de duración.

\section{Caso 2}

Primera hija de padres sanos no consanguíneos, sin antecedentes de epilepsia en familiares de primer grado, debutó a los 2 meses de edad, con CF hemiclónica derecha, de $15 \mathrm{~min}$ de duración, con paresia de Todd posterior. Las recurrencias fueron frecuentes, del tipo focal compleja y hemiclónicas de más de 10 min con al menos $5 S E$ febriles entre el 3 y 6 mes, con un examen normal post crisis.

La RM cerebral ${ }^{3}$ fueron normal, al igual que múltiples EEG standard, la cuantificación de aminoácidos en sangre, líquido cefalorraquídeo, ácidos orgánicos y ultramicroscopía de piel. A los 6 meses se solicitó estudio molecular del gen SCN1A confirmándose mutación c.5347G $>$ A (p.Ala1783Thr) en estado heterocigoto en el exón 26 del gen. El estudio molecular de ambos padres fue normal.

El uso de fenobarbital, clobazam, levetiracetam y topiramato, no logró control de crisis. A los 9 meses se inició stiripentol dosis $50 \mathrm{mg}$ $\mathrm{kp}$ día, asociado a topiramato y ácido valproico sin resultados. Entre los 12-18 meses los múltiples $S E$ y crisis frecuentes deterioraron su desarrollo perdiendo marcha, control de tronco y control cefálico. Se inició dieta cetogénica la que se asoció a una pancreatitis grave por lo que se discontinuó.

La certificación de alteración deglución y cuadros respiratorios recurrentes febriles que precipitaban los $S E$ plantearon gastrostomía lo que logró disminución de episodios febriles y mejor manejo de las crisis.

Posterior a una serie de adecuaciones de fármacos antiepilépticos el tratamiento triasociado con clobazam, topiramato y lacosamida permitió un control parcial de crisis, $\sin S E$ en 18 meses, con crisis de corta duración (segundos) 3-5 por semana que no requerían uso diazepam ni provocaban alteración en actividades de vida diaria.

\section{Caso 3}

Tercera hija, embarazo y parto normal, sin antecedentes de epilepsia en familiares de primer grado. Inició CF a los 6 meses, prolongadas, focales, clonías de hemicara y $S E$ febriles con mulitples EEG standard normales. A los 10 meses se le agregan crisis afebriles pero gatilladas por cuadros infecciosos. Posterior al año persistió con múltiples crisis afebriles hemigeneralizadas, mioclónicas, ausencias atípicas y tónicas clónicas generalizadas precipitadas por foto estimulación. Entre el primer 
y segundo año presentó $7 S E$ que requirieron ingreso a Unidad de cuidados intensivos y 10 hospitalizaciones por crisis prolongadas. La triasociación de fenobarbital, ácido valproico y levetiracetam no logró control de crisis. La RM cerebral y estudios metabólicos fueron normales.

Evolucionó con retraso de lenguaje, compromiso motor de menor cuantía y microcefalia. A la edad de 3 años se solicitó estudio molecular del gen SCN1A que confirmó la mutación c.3311C $>$ A (p.Ser1104X) exón 16 en estado heterocigoto para el gen. El estudio molecular de ambos padres fue normal. El tratamiento con topiramato, clobazam y Levetiracetam permitió controlar crisis prolongadas. Sin $S E$ desde 9 meses pero ha persistido con 5-7 crisis semanales de corta duración que no requieren uso de benzodiacepinas.

\section{Discusión}

El SD o epilepsia mioclónica severa de la infancia es una epilepsia de difícil tratamiento, infrecuente $^{12}$, que lleva a discapacidad intelectual y motora en la infancia con dependencia en la adultez. En la mayoría de los casos involucra una mutación de novo del gen del canal de sodio $\mathrm{SCN} 1 \mathrm{~A}^{13}$, mutación que fue confirmada en los casos presentados.

En nuestros pacientes pudimos constatar 2 de los 3 estadios del SD ${ }^{14,15}$. El estadio 1 o fase febril en el caso 1 y 2 se diagnosticó como CF complejas (hemiclónicas, ausencias atípicas, status febriles). La normalidad del examen neurológico y los estudios, electrofisiológicos (EEG), metabólicos (aminoácidos en sangre y LCR, ácidos orgánicos) RM cerebral y ultramicroscopía de piel retrasaron el diagnóstico en el caso 1 (18 meses), pero permitió un diagnóstico precoz en el caso 2 (6 meses de edad). La fase 2 o fase catastrófica en ambos pacientes se manifestó con convulsiones afebriles, $S E$ generalizados y parciales. Estas convulsiones motivaron múltiples esquemas de tratamiento farmacológicos que se asociaron a patología digestiva grave (hepatitis y pancreatitis). En esta fase, y en forma tardía, se diagnosticó nuestra tercera paciente cuya clínica podría se- mejar un SD like. El estadio 3 o secuelar con mejor control de crisis pero graves problemas conductuales no lo hemos experimentado aún en nuestros pacientes, pero nos mantiene alerta para realizar los cambios necesario que optimicen su calidad de vida.

En relación al tratamiento, Chiron y Du$1 a c^{16}$ muestran que el ácido valproico, las benzodiacepinas (Clobazam y clonazepam) y el topiramato son los antiepilépticos más usados en el SD, los que no fueron efectivos en nuestros pacientes y se asoció, en el caso 1 a hepatitis grave (ácido valproico). La dieta cetogénica, tratamientos no farmacológico igualmente recomendado ${ }^{11-13}$, se asoció a pancreatitis en el caso 2. El Stiripentol, droga registrada en Europa, Canadá y Japón, que actúa como modulador alostérico del receptor del ácido gama aminobutírico y recomendada para el SD, no tuvo el efecto antiepilépticos deseado en nuestro paciente (caso 2) con dosis de 40-50 mg kp día asociado a topiramato, ácido valproico y clonazepam ${ }^{17}$. Igualmente se usó el Sulthiame, que también ha sido recomendado para SD, con respuesta parcial. Es importante además destacar que la lamotrigina, carbamazepina, vigabatrina y Fenitoina exacerban la epilepsia en el SD, por lo que deben excluirse ${ }^{18}$. La fenitoína se usó frecuentemente en nuestros pacientes al llegar a urgencia, antes de tener el diagnóstico, nunca logró control de convulsiones y aumentó las mioclonías.

Otra terapia no farmacológica recomendada es la profilaxis de la fiebre e infecciones recurrentes. Nuestros pacientes tenían neumonías recurrentes, que gatillaban $S E$, lo que hizo estudiar la indemnidad de los reflejos de protección de vía aérea. Al diagnosticarse trastorno de deglución se realizó gastrostomía con fondoduplicación de Nissen lo que en ambos casos (casos 1 y 2) disminuyó los episodios de neumonías y con ello los $S E$ recurrentes.

Hattori et $\mathrm{a}^{19}$, en el año 2008 , diseñó una escala (tabla 1) que permite tener una alta sospecha diagnóstica de SD diferenciándolas en forma precoz de las CF complejas (antes del año de edad). Los 3 hitos más importantes: son inicio de CF antes de los 7 meses, más de $5 \mathrm{CF}$ en el primer año, hemiconvulsión y convulsiones prolongadas de más de 10 min de duración 
Tabla 1. Puntaje de riesgo para un tamizaje de Síndrome de Dravet

\begin{tabular}{|lc|}
\hline Factores de riesgo & Puntaje de riesgo \\
Puntaje clínico & \\
- Inicio $\geq 7$ meses & 2 \\
- $\geq 5$ crisis febriles & 3 \\
- Hemiconvulsión & 3 \\
- Crisis focal & 1 \\
- Crisis mioclónicas & 1 \\
- Crisis prolongadas & 3 \\
- Inducción con baño caliente & 2 \\
Puntaje genético & \\
- Mutación missense SCN1A & 1 \\
- Mutación truncated SCN1A & 2 \\
\hline
\end{tabular}

Fuente: Hattori et al. A screening test for the prediction of Dravet syndrome before one year of age. Epilepsia, 49 (4):626-633, 2008.

o $S E$ febriles. Nuestros pacientes, reunieron todas estas condiciones

Este diagnóstico también debe ser considerado en adultos con inicio de epilepsia refractaria en etapa de lactante, reevaluando historia clínica y estudiando mutación SCN1A.

\section{Conclusiones}

Los grandes avances en la genética nos permiten utilizar estos conocimientos en el estudio de las epilepsias e identificar epilepsias graves, difícil de reconocer en el primer estadio, permitiéndonos un manejo adecuado, innecesarias evaluaciones pre quirúrgicas, terapia antiepiléptica óptima que puede reducir las crisis y optimizar el desarrollo a largo plazo.

Agradecimientos: A Groupe Hospitalier Pitie-Salpetriere quienes realizaron los estudios genéticos correspondientes.

Potenciales conflictos de interés: Este trabajo cumple con los requisitos sobre consentimiento/asentimiento informado, comité de ética, financiamiento, estudios animales y sobre la ausencia de conflictos de intereses según corresponda.

\section{Referencias}

1.- Dravet C, Roger J, Bureau M, Dalla Bernardina M: Myoclonic epilepsies in childhood: XIIIth Epilepsy
International Symposium. In: Akimoto H, Kazamatsuri H, Seino M, Ward A, editor. Advances in Epileptology New York: Raven Press; 1982. p. 135-41.

2.- Dravet C: Les epilepsies graves de l'enfant. Vie Med 1978; 8: 543-8.

3.- Hurst DL: Epidemiology of severe myoclonic epilepsy of infancy. Epilepsia 1990; 31 (4): 397-400.

4.- Dravet $C$ : Severe myoclonic epilepsy in infants and its related syndromes. Epilepsia 2000; 41 Suppl 9: 7.

5.- Dalla Bernardina B, Dulac O, Fejerman N, et al: Early myoclonic epileptic encephalopathy (E.M.E.E.). Eur J Pediatr 1983;140 (3): 248-52.

6.- Dravet C, Bureau M, Oguni H, Fukuyama Y, Cokar O: Severe myoclonic epilepsy in infancy: Dravet syndrome. Adv Neurol 2005; 95: 71-102.

7.- Sillanpaa M, Camfield P, Camfield C, et al: Incidence of febrile seizures in Finland: prospective populationbased study. Pediatr Neurol 2008; 38 (6): 391-4.

8.- Millichap JG: The definition of febrile seizures. In: Nelson K, Ellenberg J, editors. Febrile seizures. 2nd ed. New York: Raven; 1981. p. 1-3.

9.- Oka E, Ishida S, Ohtsuka Y, Ohtahara S: Neuroepidemiological Study of Childhood Epilepsy by Application of International Classification of Epilepsies and Epileptic Syndromes (ILAE, 1989). Epilepsia 1995; 36 (7): 658-61.

10.- Claes L, Del-Favero J, Ceulemans B, Lagae L, Van Broeckhoven C, De Jonghe P: De novo mutations in the sodium-channel gene SCN1A cause severe myoclonic epilepsy of infancy. Am J Hum Genet 2001; 68 (6): 1327-32.

11.- Fujiwara T, Sugawara T, Mazaki-Miyazaki E, et al: Mutations of sodium channel alpha subunit type 1 (SCN1A) in intractable childhood epilepsies with frequent generalized tonic-clonic seizures. Brain 2003; 126 (Pt 3): 531-46.

12.- Brunklaus A, Ellis R, Reavey E, Forbes GH, Zuberi SM: Prognostic, clinical and demographic features in SCN1A mutation-positive Dravet syndrome. Brain 2012; 135 (Pt 8): 2329-36.

13.- Mulley JC, Nelson P, Guerrero S, et al: A new molecular mechanism for severe myoclonic epilepsy of infancy: exonic deletions in SCN1A. Neurology 2006; 67 (6): 1094-5.

14.- Casse-Perrot $C$, Wolf $M$, Dravet C: Neuropsychological aspects of severe myoclonic epilepsy in infancy. In: Jambaque I, Lassonde M, Dulac O, editor. Neuropsychology of Childhood Epilepsy. p 131-140 ed. New York: Kluwer Academic; 2001. p. 131-40.

15.- Camfield P, Camfield C, Nolan K: Helping families cope 
with the devastation of Dravet syndrome. Eur J Paediatr Neurol 2012; 16 Suppl 1: S9-12.

16.- Chiron C, Dulac O: The pharmacologic treatment of Dravet syndrome. Epilepsia 2011; 52 Suppl 2: 72-5.

17.- Fisher JL: The anti-convulsant stiripentol acts directly on the GABA(A) receptor as a positive allosteric modulator. Neuropharmacology 2009; 56 (1): 190-7.
18.- Korff C, Laux L, Kelley K, Goldstein J, Koh S, Nordli $D J r$ : Dravet syndrome (severe myoclonic epilepsy in infancy): a retrospective study of 16 patients. J Child Neurol 2007; 22 (2): 185-94.

19.- Hattori J, Ouchida M, Ono J, et al: A screening test for the prediction of Dravet syndrome before one year of age. Epilepsia 2008; 49 (4): 626-33. 